\title{
3.3 Age- and Sex-specific Reference Intervals for Brachial Artery Flow-mediated Dilation in Healthy Individuals and the Relation with Cardiovascular Risk Factors
}

\author{
Sophie Holder ${ }^{1,}$, Rosa Maria Bruno ${ }^{2}$, Daria Shkredova ${ }^{3}$, Andrew Thompson ${ }^{4}$, Ellen Dawson ${ }^{1}$, Helen Jones ${ }^{1}$, \\ Nicola Hopkins ${ }^{1}$, Maria Hopman ${ }^{5}$, Tom Bailey ${ }^{6}$, Jeff Coombes ${ }^{7}$, Christopher Askew ${ }^{8}$, Louise Naylor 9 \\ Andrew Maiorana ${ }^{10}$, Lorenzo Ghiadoni ${ }^{2}$, Daniel Green ${ }^{9}$, Dick Thijssen ${ }^{1,5}$ \\ ${ }^{1}$ Liverpool John Moores University, Liverpool, United Kingdom \\ ${ }^{2}$ University of Pisa, Pisa, Italy \\ ${ }^{3}$ University of British Columbia, Kelowna, Canada \\ ${ }^{4}$ University of Liverpool, Liverpool, United Kingdom \\ ${ }^{5}$ Radboud University Medical Center, Nijmegen, The Netherlands \\ ${ }^{6}$ The University of Queensland, Brisbane, Australia; University of the Sunshine Coast, Sippy Downs, Australia \\ ${ }^{7}$ The University of Queensland, Brisbane, Australia \\ ${ }^{8}$ University of the Sunshine Coast, Birtinya, Australia \\ ${ }^{9}$ The University of Western Australia, Crawley, Australia \\ ${ }^{10}$ Curtin University, Perth, Australia; Fiona Stanley Hospital, Perth, Australia
}

\begin{abstract}
Background: Assessment of endothelial function using brachial artery flow-mediated dilation (FMD) predicts future cardiovascular disease (CVD) risk. However, poor adherence to protocol guidelines and lack of reference values hinders widespread FMD use. This study established age- and sex-specific reference intervals for brachial artery FMD in healthy individuals and examined the relation with CVD risk factors.

Methods: Collected according to expert-consensus guidelines, we combined brachial artery FMD and subject characteristics/medical history from 5,362 individuals (4-84 years; 2,076 females). We first examined healthy individuals $(n=1,403$ [582 females]) to generate age-/sex-specific percentile curves. Subsequently, we included subjects with CVD risk factors but without disease (un-medicated $n=3,167$ [1,247 females], and medicated $n=792$ [247 females]). Multiple linear regression tested the relation of CVD risk factors with FMD.

Results: Healthy men showed a negative, curvilinear relation between FMD and age, whilst females revealed a linear relation that started higher, but declined at a faster rate. Age-/sex-related differences in FMD, at least partly, relate to baseline artery diameter. FMD was affected by CVD risk factors in un-medicated (e.g. systolic-/diastolic blood pressure, diabetes) and medicated subjects (e.g. dyslipidaemia). Importantly, sex mediated these effects $(p<0.05)$, with (supra) normalisation of FMD in medicated men, but not in women (except for blood pressure).

Conclusion: Sex alters the age-related decline in FMD, which is partly explained through differences in artery diameter. Sex also altered the effect of some CVD risk factors and medication on FMD. This work improves interpretation and future use of the FMD technique when strictly adhering to FMD protocol guidelines.
\end{abstract}

(c) 2019 Association for Research into Arterial Structure and Physiology. Publishing services by Atlantis Press International B.V. This is an open access article distributed under the CC BY-NC 4.0 license (http://creativecommons.org/licenses/by-nc/4.0/). 